\title{
An application of a reflection principle
}

\author{
by
Zofia Adamowicz, Leszek Aleksander Kołodziejczyk and Paweł Zbierski (Warszawa)

\begin{abstract}
We define a recursive theory which axiomatizes a class of models of $I \Delta_{0}+$ $\Omega_{3}+\neg$ exp all of which share two features: firstly, the set of $\Delta_{0}$ definable elements of the model is majorized by the set of elements definable by $\Delta_{0}$ formulae of fixed complexity; secondly, $\Sigma_{1}$ truth about the model is recursively reducible to the set of true $\Sigma_{1}$ formulae of fixed complexity.
\end{abstract}

In the present paper, we define a consistent recursive theory $T$, implying $I \Delta_{0}$ and inconsistent with $I \Delta_{0}+\exp$, which has the following two properties:

1) in every model $\mathbf{M}=T$ elements definable by $\Delta_{0}$ formulae of fixed quantifier complexity are cofinal among all $\Delta_{0}$ definable elements;

2) for every model $\mathbf{M}=T$, the set of $\Sigma_{1}$ sentences true in $\mathbf{M}$ is recursively reducible to the set of true $\Sigma_{1}$ sentences whose $\Delta_{0}$ part has fixed quantifier complexity.

Thus, $T$ axiomatizes to some extent the phenomenon of the cofinality of elements definable by $\Delta_{0}$ formulae with fixed complexity among all $\Delta_{0}$ definable elements, and of the reducibility of the set of true $\Sigma_{1}$ sentences to the set of true $\Sigma_{1}$ sentences whose complexity is fixed.

From the logical point of view, the idea behind the construction of $T$ seems to be interesting in itself. The axioms of $T$ reduce the validity of a $\Pi_{1}$ sentence $\psi$ to the validity a sentence expressing (roughly) a form of "consistency" of $\psi$. To show the consistency of $T$, we have to be able to build a model in which all "consistent" $\Pi_{1}$ sentences are true.

We construct such a model by iterating the following procedure: given a model $\mathbf{M}$ satisfying the "consistency" of the $\Pi_{1}$ sentence $\psi_{0}$, we build another model $\mathbf{M}_{0}$ satisfying $\psi_{0}$, and still satisfying the "consistency" of $\psi_{0}$. We then move on to the next $\Pi_{1}$ sentence, $\psi_{1}$. To carry on the construction,

2000 Mathematics Subject Classification: Primary 03F30.

Research of Z. Adamowicz supported in part by The State Committee for Scientific Research, Poland (KBN), grant number 5 PO3A 03720. 
we now must - if $\mathbf{M}_{0}$ satisfies the "consistency" of $\psi_{1}$ - be able to construct another model $\mathbf{M}_{1}$ satisfying $\psi_{1}$, but still satisfying $\psi_{0}$ and the "consistency" of $\psi_{0}$ and $\psi_{1}$, etc.

Thus, we need our models to have the property that "what is true is consistent". Moreover, this property has to be preserved under iteration. Therefore, what we need is in fact the " "consistency" of the set of true $\Pi_{1}$ and $\Sigma_{1}$ sentences together with the "consistency" of the set of true $\Pi_{1}$ and $\Sigma_{1}$ sentences together with the "consistency" of the set of true $\Pi_{1}$ and $\Sigma_{1}$ sentences...". To make this formal, we have to define a kind of "selfreproducing consistency statement". This is subtle since we are very close to contradicting Gödel's second incompleteness theorem.

The paper is organized as follows. Section 1 is preliminary. Section 2 discusses our basic technical tool: evaluations on sequences of terms. In Section 3, we define our "self-reproducing consistency statement", and we argue that it is a kind of reflection principle. Finally, in Section 4 we introduce the theory $T$ and prove our main results.

Acknowledgements. We would like to thank the referee for his deep insight into the paper and very valuable suggestions.

1. Preliminaries. Some notational conventions: The symbol log stands for the discrete-valued binary logarithm function; $\exp x$ is $2^{x}$. Whenever $f$ denotes a function, $f^{(k)}$ denotes $f$ iterated $k$ times. For a model $\mathbf{M}$, $\log ^{(k)}(\mathbf{M})$ (the $k$ th logarithm of $\mathbf{M}$ ) consists of those elements of $\mathbf{M}$ for which $\exp ^{(k)}$ exists. The variable $i$, possibly with indices, always ranges over elements of $\log ^{(3)}$. A "bar" (as in, say, " $\bar{x}$ ") always denotes a tuple - depending on the context, it may happen that tuples of nonstandard length are also allowed.

We adopt the coding of sets and sequences in bounded arithmetic developed in [HP]. Also the notion of length $\operatorname{lh}(\Lambda)$ of a sequence $\Lambda$ is the one defined in [HP] for bounded arithmetic. If $\Lambda=\left\langle t_{1}, \ldots, t_{l}\right\rangle$ is a sequence of length $l \in \log (\mathbf{M})$, then functions from $\Lambda$ into $\{0,1\}$ may be coded as subsets of size $\operatorname{lh}(\Lambda)$ of $\Lambda \times\{0,1\}$ (see $[\mathrm{S}]$ ). We use a somewhat different coding, letting $f: \Lambda \rightarrow\{0,1\}$ be represented by the pair $\langle\Lambda, p\rangle$, where $p$ is a function from $\{1, \ldots, l\}$ into $\{0,1\}$ - thus, an object of size $\exp l$-with $p(i)$ intended to code $f\left(t_{i}\right)$. Whenever $\Lambda$ is fixed, we may simply identify $f$ with $p$.

Our base language $L$ contains the individual constants 0,1 , and the relational symbols $+, \leq, \times,|\cdot|, \#_{2}, \#_{3}$, and $\#_{4}$.

The intuitive meaning of $|x|=y$ is that $y$ is the length of the binary representation of $x$ (equal to $\lceil\log (x+1)\rceil$ ). The $\#_{i}$ 's are to stand for the graphs of the first three smash functions: $x \#_{2} y=\exp (|x| \cdot|y|), x \#_{n+1} y=$ $\exp \left(|x| \#_{n}|y|\right)$ for $n \geq 2$. A hierarchy of functions related to the smash 
functions is defined by: $\omega_{1}(x)=x^{|x|}, \omega_{n+1}(x)=\exp \left(\omega_{n}(|x|)\right)$. Note that for any $n \geq 1, \omega_{n}(x)$ is roughly $x \#_{n+1} x$.

We assume that some appropriate Gödel numbering of $L$-formulae has been fixed; we shall identify the formulae with their Gödel numbers.

An $L$-formula $\varphi$ is in negation normal form if no quantifiers in $\varphi$ occur in the scope of a negation. $\varphi$ is $\Delta_{0}$ if all the quantifiers in $\varphi$ are bounded, i.e. of the form $\exists x \leq y . \Sigma_{1}$ and $\Pi_{1}$ formulae are defined in the natural way.

For any natural number $r$, the class $E_{r}$ consists of $\Delta_{0}$ formulae in prenex normal form which contain $r-1$ alternations of quantifier blocks, starting with an existential block, and not counting sharply bounded quantifiers $\left(^{1}\right)$. The class $U_{r}$ is defined dually. The class $\exists_{r}$ consists of $\Sigma_{1}$ formulae of the form $\exists x \psi$ where $\psi$ is $U_{r-1}$. The class $\forall_{r}$ is defined dually.

We take $I \Delta_{0}+\Omega_{3}$ to be the theory which consists of: a finite number of basic axioms relating the interpretations of the $L$-symbols to each other; the induction scheme for all $\Delta_{0}$ formulae; and an axiom stating that $\#_{4}$ is a total function (note that this is equivalent to the totality of $\omega_{3}$ ). I $\Delta_{0}+\Omega_{n}$, for $i=1,2$, is defined analogously. $I \Delta_{0}$ states only the totality of + and $\times$. $I \Delta_{0}+\exp$, on the other hand, additionally states the totality of the exp function.

$I \Delta_{0}^{*}$ is an auxiliary system which contains the basic axioms and the $\Delta_{0}$ induction scheme, but no axioms stating the totality of,$+ \times$ etc. Thus, a model of $I \Delta_{0}^{*}$ may have a greatest element. Note that (under a reasonable choice of the basic axioms), all axioms of $I \Delta_{0}^{*}$ are $\Pi_{1}$.

One benefit of working with a relational language is that defining the relativization of a formula poses no difficulties. Namely, if $\varphi$ is an $L$-formula, then $\varphi^{x}$ is defined inductively, with only the quantifier step nontrivial: $(\exists y \psi)^{x}:=\exists y \leq x \psi^{x}$.

The language $L_{T}$ is an extension of $L$ obtained by adding function symbols $s^{\varphi}$ for all $L$-formulae $\varphi$ in negation normal form which begin with an existential quantifier. The intention is that the symbol $s^{\varphi}$ stands for a Skolem function for the first existential quantifier in $\varphi$. That is, given an $L$-formula $\varphi(\bar{x})=\exists y \psi(\bar{x}, y)$ in negation normal form, $s^{\varphi}$ is a function symbol of arity $1+\operatorname{lh}(\bar{x})$, and $s^{\varphi}(\bar{t})$ is intended to be some $y$ which satisfies $\psi(\bar{t}, y)$, if such a $y$ exists.

Whenever we speak of a formula $\varphi(\bar{t})$, it is assumed that $\varphi(\bar{x})$ itself is an $L$-formula, although the terms $\bar{t}$ do not have to be terms of $L$.

We have to encode the language $L_{T}$ in arithmetic. We use numbers divisible by 3 to enumerate terms of the form $s^{\varphi}(\bar{t})$, numbers congruent to

$\left({ }^{1}\right)$ The notion of sharply bounded quantifier is an obvious variant of the one known from functional languages for bounded arithmetic, e.g. in $\forall x \forall y \leq x \exists z \leq x \quad((y=|x| \Rightarrow$ $z=y) \wedge \ldots$ ) the quantifier $\forall z$ is sharply bounded. 
$1(\bmod 3)$ for a special enumeration of numerals, and numbers congruent to $2(\bmod 3)$ to enumerate some additional terms. In more detail: we let the number $3\langle\varphi(\bar{x}), \bar{t}\rangle$ correspond to $s^{\varphi}(\bar{t})$; we let $3 k+1$ correspond to a numeral for $k(3 k+1$ will be referred to as $\underline{k})$; finally, we let $3 k+2$ correspond to a special term $s_{k}$ (the role of the $s_{k}$ 's is explained in clause (v) of Definition 2.3 below). We also code $\varphi(\bar{t})$ by the ordered pair $\langle\varphi(\bar{x}), \bar{t}\rangle$.

From now on, we identify the terms of $L_{T}$ with their numbers.

The models $\mathbf{M}$ we work with are - unless explicitly stated or obvious from the context that this is not the case-assumed to be nonstandard countable models of $I \Delta_{0}+\Omega_{3}$.

We shall consider various sequences of closed terms. About such a sequence $\Lambda$ we shall always assume that if a term of the form $s^{\varphi}(\bar{t})$ appears in $\Lambda$, then all terms in $\bar{t}$ also do, and moreover, that their indices in $\Lambda$ are smaller than the index of $s^{\varphi}(\bar{t})$. Also, whenever dealing with a sequence $\Lambda$ and a model $\mathbf{M}$, we shall assume that $\operatorname{lh}(\Lambda)$ is in $\log (\mathbf{M})$.

Given a sequence of terms $\Lambda$, let the collection $\mathcal{A}(\Lambda)$ of atomic sentences over $\Lambda$ consist of all sentences obtained by substituting terms from $\Lambda$ for variables in atomic formulae of $L$. Observe that there is a standard polynomial $\pi(n)$ such that $\operatorname{lh}(\mathcal{A}(\Lambda)) \leq \pi(\operatorname{lh}(\Lambda))$. Let us fix some such $\pi$.

Some more notation: if $\mathcal{F}$ is a class of formulae, the symbol $\mathcal{F}(\mathbf{M})$ denotes the family of all $\mathcal{F}$-definable elements of $\mathbf{M}$, while $\mathbf{M}^{\mathcal{F}}$ denotes the set of $\mathcal{F}$-sentences true in $\mathbf{M}$.

Finally, let us recall some relevant facts about universal formulae. Firstly, in $I \Delta_{0}+\exp$ there is a $\Sigma_{1}$ universal formula Sat for $\Delta_{0}$. Thus, Sat is $\Sigma_{1}$, and for any $\mathbf{M} \models I \Delta_{0}+\exp , \varphi \in \mathbf{M}$ a $\Delta_{0}$ formula,

$$
\mathbf{M}=\operatorname{Sat}(\varphi) \quad \text { iff } \quad \mathbf{M}=\varphi .
$$

Secondly, in $I \Delta_{0}+\Omega_{3}$ there is an $\exists_{r}$ universal formula Sat ${ }_{r}$ for $\exists_{r}$, for each $r \in \omega$. Sat $r$ can obviously also be used as a universal formula for $E_{r}$, and additionally, if we limit our attention to the truth of $E_{r}$ formulae smaller than some $a$ with parameters smaller than some $b$, then the initial existential quantifier in $\mathrm{Sat}_{r}$ can also be bounded (thus giving an " $E_{r}$ formula with a parameter": call this formula $\left.\mathrm{Sat}_{E_{r}}\right)$.

2. Evaluations and evaluation models. Let $p: \mathcal{A}(\Lambda) \rightarrow\{0,1\}$ map every axiom of equality in $\mathcal{A}(\Lambda)$ to 1 . We call such a $p$ an evaluation on $\Lambda$, since we may think of $p$ as assigning a logical value to sentences in $\mathcal{A}(\Lambda)$ (see also [A1]-[A3], [AZ1], [AZ2], [S]). Of course, $p$ can be uniquely extended to all boolean combinations of sentences in $\mathcal{A}(\Lambda)$ in the routine way.

Note in passing that any evaluation on $\Lambda$ is an object of size at most $\exp (\operatorname{lh}(\mathcal{A}(\Lambda)))$ and thus at most $\exp (\pi(\operatorname{lh}(\Lambda)))$. 
For $\varphi(\bar{x})$ in negation normal form and $\bar{t} \in \Lambda$, we define the notion of $\Lambda$ being good enough (g.e.) for $\langle\varphi, \bar{t}\rangle$ by induction on $\varphi$. First, $\Lambda$ is always g.e. for $\langle\varphi, \bar{t}\rangle$ if $\varphi$ is open; $\Lambda$ is g.e. for $\left\langle\varphi_{1} \vee \varphi_{2}, \bar{t}\right\rangle$ iff it is g.e. for $\left\langle\varphi_{1}, \bar{t}\right\rangle$ and $\left\langle\varphi_{2}, \bar{t}\right\rangle$, and similarly for conjunctions. If $\varphi$ is $\exists y \varphi^{\prime}(\bar{x}, y)$, then $\Lambda$ is g.e. for $\langle\varphi, \bar{t}\rangle$ if $s^{\varphi}(\bar{t}) \in \Lambda$ and $\Lambda$ is g.e. for $\left\langle\varphi^{\prime}, \bar{t}^{\urcorner} s^{\varphi}(\bar{t})\right\rangle$. Finally, if $\varphi$ is $\forall y \widetilde{\varphi}(\bar{x}, y)$,

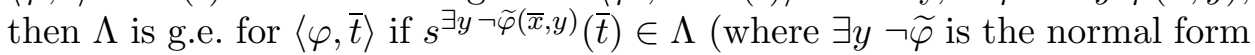

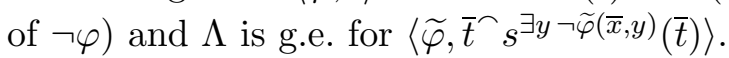

The idea is that $\Lambda$ is g.e. for $\langle\varphi, \bar{t}\rangle$ if it contains enough appropriate Skolem terms so that assigning a logical value to $\varphi(\bar{t})$ based on an evaluation on $\Lambda$ makes sense.

Definition 2.1. Let $\bar{t} \in \Lambda$. We define the relation $p \models \varphi(\bar{t})$ for $\varphi(\bar{x})$ in negation normal form by induction:

(i) $p=\varphi(\bar{t})$ iff $p(\varphi(\bar{t}))=1$ for $\varphi(\bar{t})$ open;

(ii) the relation $p \models \varphi$ behaves in the natural way with respect to conjunctions and disjunctions;

(iii) if $\varphi$ is $\exists y \varphi^{\prime}(\bar{x}, y)$, then $p \models \varphi(\bar{t})$ iff $\Lambda$ is g.e. for $\langle\varphi, \bar{t}\rangle$ and $p=$ $\varphi^{\prime}\left(\bar{t}, s^{\varphi}(\bar{t})\right)$,

(iv) if $\varphi$ is $\forall y \widetilde{\varphi}(\bar{x}, y)$, then $p \models \varphi(\bar{t})$ iff for all $t \in \Lambda$ such that $\Lambda$ is g.e. for $\langle\widetilde{\varphi}, \bar{t}\urcorner t\rangle, p \mid=\widetilde{\varphi}(\bar{t}, t)$.

We will be especially interested in the case where $\Lambda$ is one of a number of canonical sequences of terms. To define these, let $K(i)$ be the unique function satisfying $K(0)=1$ and $K(i+1)=c \cdot \exp (i) \cdot K(i)^{i}$, where $c$ is an appropriately large standard integer. Note that for any $i, K(i) \in \log$, as for almost all $i, K(i) \leq \exp \left(i^{i}\right)$, and $i^{i}$ is always in $\log ^{(2)}$, since we have:

REMARK 2.2. In any model of $I \Delta_{0}+\Omega_{3}, \log$ is closed under $\omega_{2}, \log ^{(2)}$ is closed under $\omega_{1}, \log ^{(3)}$ is closed under multiplication, and $\log ^{(4)}$ is closed under addition.

Moreover, the sequence $\left\langle K(i): i \in \log ^{(3)}\right\rangle$ is cofinal in log.

The notion of canonical sequence of rank $i, \Lambda_{i}$, is now defined by induction. $\Lambda_{i+1}$ is the smallest sequence $\Lambda$ such that:

- for any $j \leq i+1, \Lambda$ contains the term $s^{j}$ and is good for $\left\langle\exp ^{(3)}(x)\right.$ $\left.=y, \underline{j} s_{j}\right\rangle$

- for any $a \leq K(i), \Lambda$ contains the numeral $\underline{a}$, and if $\exp a \leq \exp ^{(3)}(i+1)$, then $\Lambda$ is g.e. for $\langle\exists \exp x, \underline{a}\rangle$;

- for any formula $\varphi<\exp i$ of the form $\psi^{t}$ or $\exists x \leq t \psi^{x}$ (where $t \in$ $\Lambda_{i} \cup\left\{s_{i+1}\right\}$ ), and any $\bar{t} \in \Lambda_{i}, \Lambda$ is g.e. for $\langle\varphi, \bar{t}\rangle$.

Observe that if $c$ is chosen large enough, then $\operatorname{lh}\left(\Lambda_{i}\right) \leq K(i)$ for all $i$ (since a formula smaller than $\exp i$ contains at most $i$ quantifiers). 
Some particularly well-behaved evaluations on $\Lambda_{i}$ will be called evaluations of rank $i$ (we let $\mathcal{A}_{i}$ stand for $\mathcal{A}\left(\Lambda_{i}\right)$ ):

Definition 2.3. A function $p: \mathcal{A}_{i} \rightarrow\{0,1\}$ is called an evaluation of rank $i$ if the following holds:

(i) for every $\varphi(\bar{x})<\exp i$ and every $\bar{t} \in \Lambda_{i}$ of appropriate length, if $\Lambda_{i}$ is g.e. for $\langle\varphi, \bar{t}\rangle$, then for all $j \leq i$,

$$
p \models \varphi(\bar{t})^{s_{j}} \quad \text { or } \quad p \models \neg \varphi(\bar{t})^{s_{j}} ;
$$

(ii) if $\varphi<\exp (i)$ is an axiom of $I \Delta_{0}^{*}$, then assuming $\Lambda_{i}$ is g.e. for $\langle\varphi, \emptyset\rangle$, $p=\varphi$;

(iii) $p=(\underline{0}=0 \wedge \underline{1}=1)$, and given any $\underline{a}, \underline{b} \in \Lambda_{i}$ : if $\underline{a+b} \in \Lambda_{i}$, then $p=(\underline{a}+\underline{b}=\underline{a+b})$, and similarly for the other symbols of $L$;

(iv) for all $\underline{a} \in \Lambda_{i}$ such that $\exp a \leq \exp ^{(3)}(i+1), p=\exists \exp \underline{a}$;

(v) for all $j \leq i, p=s_{j}=\exp ^{(3)}(j)$.

We let " $p \in \mathcal{E}_{i}$ " stand for " $p$ is an evaluation of rank $i$ ". This is a slight abuse of notation, since the code for the set of evaluations of rank $i$ might be too large to be an element of the model.

We claim that both " $p \models \varphi$ " (for $p$ an evaluation on $\Lambda_{i}$ ) and " $p \in \mathcal{E}_{i}$ " are $\Delta_{0}$ definable with an appropriately large parameter (and thus $\Delta_{1}$ definable).

To see whether an evaluation $p$ on $\Lambda_{i}$ sets $\varphi$ to "True" (i.e. whether $p=\varphi$ ), we need to deal with sets $V_{0}, \ldots, V_{r}$, where $V_{l}$ is the set of values given by $p$ to the $l$ th subformula of $\varphi$ under all relevant substitutions of terms in $\Lambda_{i}$ for the free variables in that subformula. Since there are at most $\log \varphi$ variables in any subformula of $\varphi$, the number of possible substitutions is not greater than $K(i)^{\log \varphi}$, and hence $V_{l} \leq \exp \left(K(i)^{\log \varphi}\right)$. Again, there can be no more than $\log \varphi$ subformulae of $\varphi$. Thus, the sequence $\left\langle V_{0}, \ldots, V_{r}\right\rangle$ is at most $\log \varphi$-long, so its code is at $\operatorname{most} \exp \left(\log \varphi \cdot K(i)^{\log \varphi}\right)$. This is the largest object relevant to the truth value given to $\varphi$ by $\psi$, which shows that " $p=\varphi$ " is indeed $\Delta_{0}$ definable with a parameter.

To see whether an evaluation $p$ on $\Lambda_{i}$ is in $\mathcal{E}_{i}$, we have to check what truth value it assigns to a number of formulae $\varphi$ smaller than $\exp (i)$. With some additional work, one may verify that all objects we need to consider are smaller than $\omega_{3}\left(\exp ^{(3)}(i)\right)$, which implies that also " $p \in \mathcal{E}_{i}$ " is $\Delta_{0}$ definable with a parameter.

We let $\operatorname{True}(p, i, \varphi)$ be a $\Delta_{1}$ formula which says " $p \in \mathcal{E}_{i}$ and $p \models \varphi$ ".

Definition 2.4. If $p_{1} \in \mathcal{E}_{i_{1}}$ and $p_{2} \in \mathcal{E}_{i_{2}}$ with $i_{1} \leq i_{2}$, we say that $p_{2}$ extends $p_{1}$ if $p_{1} \subseteq p_{2}$.

The following proposition lists some "conservativity" relationships between evaluations one of which extends the other. The proofs are simple inductive arguments. 
Proposition 2.5. Let $p_{2} \in \mathcal{E}_{i_{2}}$ extend $p_{1} \in \mathcal{E}_{i_{1}}$. Then:

(1) if $\Lambda_{i_{1}}$ is g.e. for $\langle\varphi, \bar{t}\rangle$ and $j \leq i_{1}$, then $p_{1}=\varphi(\bar{t})^{s_{j}}$ iff $p_{2}=\varphi(\bar{t})^{s_{j}}$;

(2) if $\varphi(\bar{x})$ is an open formula and $\bar{t} \in \Lambda_{i_{1}}$, then $p_{1}=\varphi(\bar{t})$ iff $p_{2} \models \varphi(\bar{t})$;

(3) if $\Lambda_{i_{1}}$ is g.e. for $\langle\varphi, \bar{t}\rangle$, then $p_{2} \models \varphi(\bar{t})$ implies $p_{1}=\varphi(\bar{t})$.

Another simple fact about evaluations is:

Proposition 2.6. Let $p \in \mathcal{E}_{i}$ and let $i^{\prime} \leq i$. Then $\left(p \nmid \mathcal{A}_{i^{\prime}}\right) \in \mathcal{E}_{i^{\prime}}$.

The importance of evaluations consists in the fact that they make possible the construction of models for $I \Delta_{0}+\Omega_{3}$. More precisely, such a model is determined by an ascending chain of evaluations whose ranks are cofinal in $\log ^{(3)}$ (note that by Remark 2.2, in a model of $I \Delta_{0}+\Omega_{3}$ the third logarithm has no last element).

Let $P=\left\langle p_{n}: n \in \omega\right\rangle$ be such a chain. If $\bar{t}$ is a tuple of terms of $L_{T}$ and $\varphi(\bar{t})$ is open, then almost all $p_{n}$ 's agree at the logical value of $\varphi(\bar{t})$. So, we may define $P=\varphi(\bar{t})$ by

$$
p_{n}=\varphi(\bar{t}) \quad \text { for almost all } n \text {. }
$$

We define the relation $={ }_{P}$ between terms in $L_{T}$ by

$$
t_{1}={ }_{P} t_{2} \quad \text { iff } \quad P=\left(t_{1}=t_{2}\right) .
$$

Since the $p_{n}$ 's are evaluations, $=_{P}$ is an equivalence relation and a congruence with respect to the relations of $L$. Let $\mathbf{M}_{0}[P]$ be the model whose universe is the set of $={ }_{P}$-equivalence classes and whose relations are defined by

$$
\left[t_{1}\right]+\left[t_{2}\right]=\left[t_{3}\right] \quad \text { iff } \quad P \models\left(t_{1}+t_{2}=t_{3}\right) \text { etc. }
$$

Clearly, we have

$$
\mathbf{M}_{0}[P] \models \varphi([\bar{t}]) \quad \text { iff } \quad P \models \varphi(\bar{t})
$$

for any open $\varphi$. If we introduce the more general relation $P \models \varphi(\bar{t})$, for $\varphi$ not necessarily open, by the same clause as above, then induction yields

$$
P \mid=\varphi(\bar{t}) \quad \text { implies } \quad \mathbf{M}_{0}[P] \mid=\varphi([\bar{t}]) .
$$

The converse implication will not hold generally unless we adopt a more restrictive definition of evaluation which is not needed here.

The next lemma and corollary show that the numeral $\underline{a}$ may be treated as a name for the ath element of $\mathbf{M}_{0}[P]$.

Lemma 2.7. Let $p \in \mathcal{E}_{i}$. If for a term $t \in L_{T}, p \models(t \leq \underline{a})$, then there is $b \leq$ a such that $p=(t=\underline{b})$. Moreover, if $\varphi$ is an open formula and $\underline{\bar{a}}$ is a tuple of numerals for numbers less than or equal to $K(i-1)$, then $\varphi(\bar{a})$ implies $p=\varphi(\underline{\bar{a}})$.

Proof. We may assume that our formalization of $I \Delta_{0}^{*}$ contains axioms such as: $\forall x(x \leq 0 \Rightarrow x=0), \forall x \forall y(x \leq y+1 \Rightarrow x=y+1 \vee x \leq y)$, 
$\forall x(x+0=x), \forall x \forall y((x+(y+1)=(x+y)+1)$ and similar axioms for the other symbols of $L$.

The first part of the lemma is proved by induction on $a \leq K(i-1)$. For $a=0, p \models(\underline{0}=0)$, so $p \models(t \leq \underline{0})$ implies $p \models(t \leq 0)$, hence $p \models(t=0)$ by the appropriate axiom, hence $p=(t=\underline{0})$. Assume that the assertion holds for $a$ and that $p=(t \leq \underline{a+1})$. Then, since $p=(\underline{a+1}=\underline{a}+1)$, we get either $p=(t=\underline{a+1})$, or $p \models(t \leq \underline{a})$, in which case we use the inductive assumption to get $p=(t=\underline{b})$ for some $b \leq a$.

For the "moreover" part, first prove $p=\left(\underline{a_{1}}+\underline{a_{2}}=\underline{a_{1}+a_{2}}\right)$ (assuming $\left.a_{1}+a_{2} \leq K(i-1)\right)$ by induction, using appropriate axioms for the induction base and induction step. Then proceed similarly with $p=\left(a_{1} \cdot \underline{a_{2}}=a_{1} \cdot a_{2}\right)$ (again, assuming $i$ is large enough) and the remaining symbols of $\bar{L}$, and pass through boolean combinations to obtain the assertion.

Corollary 2.8. The mapping $a \mapsto[\underline{a}]($ for $a \in \log (\mathbf{M}))$ is an isomorphism between $\log (\mathbf{M})$ and an initial segment $I$ of $\mathbf{M}_{0}[P]$.

Proof. It suffices to observe that if $P=\left\langle P_{n}: n \in \omega\right\rangle$ where $p_{n} \in \mathcal{E}_{i_{n}}$, then for any tuple $\bar{a} \in \log$, the maximal element of $\bar{a}$ is smaller than $K\left(i_{n}-1\right)$ for almost all $n$, so we may apply Lemma 2.7.

By clause (iv) of Definition $2.3, I \subseteq \log \left(\mathbf{M}_{0}[P]\right)$. Let $\mathbf{M}[P]$ be the initial segment of $\mathbf{M}_{0}[P]$ generated by $\exp (I)$. If we identify $I$ with $\log (\mathbf{M})$, we obtain:

Corollary 2.9. $\log (\mathbf{M})=\log (\mathbf{M}[P])$. Thus, more generally, $\log ^{(n)}(\mathbf{M})$ $=\log ^{(n)}(\mathbf{M}[P])$ for all $n \geq 1$.

We also have:

Corollary 2.10. If $\varphi(\bar{x})$ is a $\Pi_{1}$ formula and $P=\varphi(\bar{t})$, then $\mathbf{M}[P] \mid=$ $\varphi([\bar{t}])$.

We close this section with a theorem on evaluation models (i.e. models of the form $\mathbf{M}[P]$ ) which will play a key role later on.

Theorem 2.11. Let $\mathbf{M}$ be a countable model of $I \Delta_{0}+\Omega_{3}+B \Sigma_{1}$. Assume that $\mathcal{F}$ is a set of standard $L$-formulae,

$$
\mathcal{F}=\left\{\theta_{n}\left(x_{1}, \ldots, x_{r}\right): n \in \omega\right\},
$$

and is a subset of a set

$$
\left\{\theta_{l}\left(x_{1}, \ldots, x_{r}\right): l \in \log ^{(3+k)}(\mathbf{M})\right\}
$$

(for some $k \in \omega$ ) which is $\Delta_{1}$ definable in $\mathbf{M}$ and satisfies

$$
\forall i \exists p \in \mathcal{E}_{i} \forall l, l_{1}, \ldots, l_{r}<\log ^{(k)}(i) p \models \theta_{l}\left(\underline{l_{1}}, \ldots, \underline{l_{r}}\right) .
$$

Then there exists an increasing and cofinal sequence $P=\left\langle p_{n}: n \in \omega\right\rangle$ 
of evaluations such that $P=\varphi\left(\underline{l_{1}}, \ldots, \underline{l_{r}}\right)$ for each $\varphi \in \mathcal{F}, \underline{l_{1}}, \ldots, \underline{l_{r}} \in$ $\log ^{(3+k)}(\mathbf{M})$, and the model $\mathbf{M}[P]$ satisfies $I \Delta_{0}+\Omega_{3}$.

In particular, for any $n$ such that $\theta_{n}$ is $\Pi_{1}, \mathbf{M}[P] \models \theta_{n}\left(l_{1}, \ldots, l_{r}\right)$ for each $l_{1}, \ldots, l_{r} \in \log ^{(3+k)}(\mathbf{M}), n \in \omega$.

Proof. Let us introduce the following convention: every evaluation $p$ of rank $i$ appearing in this proof satisfies $p \models \theta_{l}\left(\underline{l_{1}}, \ldots, \underline{l_{r}}\right)$ for all $l, l_{1}, \ldots, l_{r}<$ $\log ^{(k)} i$.

Let $i_{1}<i_{2}<\ldots$ be cofinal in $\log ^{(3)}(\mathbf{M})$. We shall define a sequence $P=\left\langle p_{n}: n \in \omega\right\rangle$ such that $p_{n} \in \mathcal{E}_{i_{n}}$.

$P$ is defined by induction as follows. Suppose that at a given step $n$ we already have evaluations $p_{1} \subseteq \ldots \subseteq p_{n}$ such that $p_{1} \in \mathcal{E}_{i_{1}}, \ldots, p_{n} \in \mathcal{E}_{i_{n}}$ satisfying the inductive condition

$$
\forall i>i_{n} \exists p \in \mathcal{E}_{i}\left[p_{n} \subseteq p\right] .
$$

Note that at the initial step the validity of the inductive condition is ensured by the assumption of the theorem.

We claim that it follows by $B \Sigma_{1}$ that

$$
\exists p_{n+1} \in \mathcal{E}_{i_{n+1}} \forall i>i_{n+1} \exists p \in \mathcal{E}_{i}\left[p_{n} \subseteq p_{n+1} \subseteq p\right] .
$$

Indeed, assume $(* *)$ fails. Then for any $\widetilde{p} \in \mathcal{E}_{i_{n+1}}$ extending $p_{n}$ there exists $i(\widetilde{p})>i_{n+1}$ for which there is no evaluation $p \in \mathcal{E}_{i}$ extending $\widetilde{p}$. Now, all $\widetilde{p}$ s are bounded by $\exp \left(\pi\left(K\left(i_{n+1}\right)\right)\right.$. Thus, we may use $B \Sigma_{1}$ to find a common bound $i$ for all the $i(\widetilde{p})$ 's. It follows that there is no $p \in \mathcal{E}_{i}$ extending any of the $i(\widetilde{p})$ 's. On the other hand, by $(*)$ there is some $p \in \mathcal{E}_{i}$ extending $p_{n}$. $\operatorname{But}\left(p\left\lceil\mathcal{A}_{i_{n+1}}\right) \in \mathcal{E}_{i_{n+1}}\right.$, and $p_{n} \subseteq\left(p\left\lceil\mathcal{A}_{i_{n+1}}\right) \subseteq p\right.$, a contradiction. Hence, (**) must hold and the claim is proved.

Clearly, the evaluation $p_{n+1}$ given by $(* *)$ satisfies the inductive condition at stage $n+1$.

Now let $P=\left\langle p_{n}: n \in \omega\right\rangle$. Obviously $P$ is increasing and cofinal. Since all the axioms of $I \Delta_{0}^{*}$ are $\Pi_{1}$ we infer from Corollary 2.10 that

$$
\mathbf{M}[P] \models I \Delta_{0}^{*} .
$$

On the other hand, the set $\left\{\exp ^{(3)}(i): i \in \log ^{(3)}(\mathbf{M})\right\}$ is cofinal in both $\mathbf{M}$ and $\mathbf{M}[P]$ (cf. Corollary 2.9). Since $\mathbf{M}=\Omega_{3}$, we infer in view of Corollary 2.9 that $\mathbf{M}[P] \models \Omega_{3}$. Consequently, $\mathbf{M}[P] \models I \Delta_{0}+\Omega_{3}$ since obviously $I \Delta_{0}^{*}+\Omega_{3}$ implies $I \Delta_{0}+\Omega_{3}$. This completes the proof of the theorem.

REMARK 2.12. To keep the formulation of the above theorem reasonably concise, we have stated its assumptions in a relatively simple way. It is clear, however, that appropriate variants of the theorem would also be true if the assumptions were modified in one or more of the following ways: 
- in (\#), $\forall i \exists p \in \mathcal{E}_{i}(\ldots)$ could be replaced by $\forall^{\infty} i \exists p \in \mathcal{E}_{i}(\ldots)$;

- also in $(\#), \forall l, l_{1}, \ldots, l_{r}<\log ^{(k)}(i)$ could be replaced by $\forall l, l_{1}, \ldots, l_{r}<$ $\left(\log ^{(k)}(i)\right) / r$ (for any standard $r$ ), as long as $\log ^{(3+k)}$ is closed under addition;

- $\mathcal{F}$ could be extended by adding finitely many formulae of the form $\varphi(\bar{t})$ evaluated to "True" by almost all of the $p$ 's given by (\#).

In what follows, we will sometimes speak of using "Theorem 2.11" when some such variant is actually meant.

3. The principle $\tau$. The present section introduces a consistent sentence $\tau$ which is a kind of reflection principle (mentioned in the title). We begin by formulating some preservation properties of evaluations.

For a $\Sigma_{1}$ sentence $\Phi$ of the form $\exists x \phi^{x}$ let $\Gamma_{\Phi}(p, i)$ be the formula

$$
\begin{aligned}
\Phi<\exp (i-1) \Rightarrow(\forall j & \leq i\left(\exists x \leq \exp ^{(3)}(j) \phi^{x} \Rightarrow \operatorname{True}\left(p, i, \exists x \leq s_{j} \phi^{x}\right)\right) \\
& \left.\wedge\left(\forall x \leq \exp ^{(3)}(i) \neg \phi^{x} \Rightarrow \operatorname{True}\left(p, i, \forall x \neg \phi^{x}\right)\right)\right),
\end{aligned}
$$

and, for a fixed sufficiently large $m$ which depends on some further constructions but could be specified in advance, let $\Gamma_{m}(p, i)$ be the formula

$$
\begin{aligned}
\forall \psi<i, \psi \in & \exists_{m} \forall j \leq i \forall \underline{a_{1}}, \ldots, \underline{a_{r}} \in \Lambda_{i} \\
& \left(\operatorname{Sat}_{m}\left(\psi^{\exp ^{(3)}(j)}\left(a_{1}, \ldots, a_{r}\right)\right) \Rightarrow \operatorname{True}\left(p, i, \psi^{s_{j}}\left(\underline{a}_{1}, \ldots, \underline{a}_{r}\right)\right)\right) .
\end{aligned}
$$

Intuitively, $\Gamma_{\Phi}(p, i)$ says " $p$ preserves the size of a witness for $\Phi=\exists x \phi^{x}$, and disallows witnesses of size greater than $\exp ^{(3)}(i)$ ", while $\Gamma_{m}(p, i)$ says " $p$ preserves the restrictions $\psi^{\exp ^{(3)}(j)}$, for $j \leq i$, of all $\exists_{m}$ sentences smaller than $i "$.

Arguments similar to those in the previous section show that both $\Gamma_{\Phi}$ and $\Gamma_{m}$ are $\Delta_{0}$ with a parameter (and hence $\Delta_{1}$ ), as they make no reference to objects greater than $\omega_{3}\left(\exp ^{(3)}(i)\right)$.

We will now define some (possibly nonstandard) sentences $\tau_{j, j_{1}}$ for $j, j_{1} \in$ $\log ^{(4)}$ (whenever the variables $j, j_{1}$ appear as indices in $\tau_{j, j_{1}}$, they are assumed to range over $\left.\log ^{(4)}\right)$. The definition is by induction on $j_{1}$. Let $\tau_{j, 0}$ be

$$
\left(\exists p \in \mathcal{E}_{\exp \underline{0}}\left\{\Gamma_{m}(p, \exp \underline{0}) \wedge \bigwedge_{\Phi \leq j} \Gamma_{\Phi}(p, \exp \underline{0})\right\}\right)^{\exp (4)(2 \cdot \underline{0})}
$$

and let $\tau_{j, j_{1}+1}$ be

$$
\begin{aligned}
& \left(\exists p \in \mathcal { E } _ { \operatorname { e x p } ( \underline { j _ { 1 } + 1 } ) } \left\{\Gamma_{m}\left(p, \exp \left(\underline{j_{1}+1}\right)\right) \wedge \bigwedge_{\Phi \leq j} \Gamma_{\Phi}\left(p, \exp \left(\underline{j_{1}+1}\right)\right)\right.\right. \\
& \left.\wedge \bigwedge_{l, l_{1}<\left(j_{1}+1\right) / 2} \operatorname{True}\left(p, \exp \left(\underline{j_{1}+1}\right), \underline{\left.\tau_{l, l_{1}}\right)}\right\}\right)^{\exp (4)\left(2 \underline{\left(j_{1}+1\right)}\right)} .
\end{aligned}
$$


If the definition of $\tau_{j, j_{1}}$ is to make sense, an evaluation of rank exp $j_{1}$ should be able to decide the truth value of $\tau_{l, l_{1}}$ for $l, l_{1}<j_{1} / 2$. To check that this is so, let $\varphi_{j, j_{1}}(z, \bar{x})$ stand for

$$
\begin{aligned}
\left(\exists y \in \mathcal { E } _ { \operatorname { e x p } z } \left\{\Gamma_{m}(y, \exp z)\right.\right. & \wedge \bigwedge_{\Phi \leq j} \Gamma_{\Phi}(y, \exp z) \\
& \left.\left.\wedge \bigwedge_{l, l_{1}<j_{1} / 2} \operatorname{True}\left(y, \exp z, x_{l, l_{1}}\right)\right\}\right)^{\exp ^{(4)}(2 z)},
\end{aligned}
$$

and $\bar{t}_{j_{1}}$ stand for $\left\langle\underline{\tau_{l, l_{1}}}: l, l_{1}<j_{1} / 2\right\rangle$.

Observe that $\tau_{j, j_{1}}$ is $\varphi_{j, j_{1}}\left(j_{1}, \bar{t}_{j_{1}}\right)$. Therefore, it is enough to check that for any $j_{1}, \varphi_{j_{1} / 2, j_{1} / 2}$ is smaller than $\exp \left(\exp \left(j_{1}\right)-1\right)$ and that $\bar{t}_{j_{1} / 2} \in \Lambda_{\exp \left(j_{1}\right)-1}$.

To see the former, note that given any $j_{1}$, a code for $\varphi_{j_{1}, j_{1}}$ is about $j_{1}^{j_{1}^{2}}$ (a precise bound on $\varphi_{j_{1}, j_{1}}$ depends on the details of how we code the syntax, esp. the variables, but the main ingredient of $\varphi_{j_{1}, j_{1}}$ is a $\left(j_{1}^{2} / 4\right)$-long conjunction of formulae whose codes will not much exceed the code for the $\left(j_{1}^{2} / 4\right)$ th variable, which in turn may be around $\left.j_{1}^{2}\right)$. So for us it suffices if $\left(j_{1} / 2\right)^{j_{1}^{2} / 4}$ is smaller than $\exp \left(\exp \left(j_{1}\right)-1\right)$, which is clearly always the case.

To see that $\bar{t}_{j_{1} / 2} \in \Lambda_{\exp \left(j_{1}\right)-1}$, we only need to check that for all $j_{1}$, $\tau_{j_{1} / 4, j_{1} / 4}$ is smaller than $K\left(\exp \left(j_{1}\right)-1\right)$. But for any $j_{1}$, the size of $\tau_{j_{1}, j_{1}}$ can be bounded by roughly $j_{1}^{j_{1}^{2}}$ (the code for $\varphi_{j_{1}, j_{1}}$ ) times the code for the $\left(j_{1}^{2} / 4\right)$-long sequence of the $\tau_{l, l_{1}}$ 's (for $l, l_{1}<j_{1} / 2$ ). This sequence will have a code smaller than $\left(3 \cdot \tau_{j_{1} / 2, j_{1} / 2}\right)^{j_{1}^{2} / 4}$. Using the fact that $K(i+1)>c K(i)^{i}$ for some large standard $c$, it is easy to verify that $K\left(\exp \left(j_{1}\right)-1\right)$ is more than $\tau_{j_{1}, j_{1}}$ (not to mention $\tau_{j_{1} / 4, j_{1} / 4}$ ).

In addition to the $\tau_{j, j_{1}}$ 's we also define, for any $j \in \log ^{(4)}$, a formula $\tau_{j}\left(j_{1}\right)$ with $j_{1}$ as a free variable:

$$
\begin{aligned}
\left(\exists p \in \mathcal { E } _ { \operatorname { e x p } j _ { 1 } } \left\{\Gamma_{m}\left(p, \exp j_{1}\right)\right.\right. & \wedge \bigwedge_{\Phi \leq j} \Gamma_{\Phi}\left(p, \exp j_{1}\right) \\
& \left.\left.\wedge \forall l, l_{1}<j_{1} / 2 \forall x\left(x=\tau_{l, l_{1}} \Rightarrow \operatorname{True}\left(p, \exp j_{1}, x\right)\right)\right\}\right)^{\exp ^{(4)}\left(2 j_{1}\right)},
\end{aligned}
$$

where $x=\tau_{l, l_{1}}$ is an abbreviation for the inductive definition of $\tau_{l, l_{1}}$ with $l$ and $l_{1}$ as parameters. Note that although the $\tau_{j}(\cdot)$ 's are in general again nonstandard, $\tau_{n}(\cdot)$ is a standard formula for any standard $n$.

Note also that $\exp ^{(4)}\left(2 j_{1}\right)$ is not less than $\omega_{3}\left(\exp ^{(4)}\left(j_{1}\right)\right)$ - the greatest element we possibly need to access in order to check whether a given $p \in$ $\mathcal{E}_{\exp j_{1}}$ satisfies all the conditions required in $\tau_{j, j_{1}}$ or $\tau_{j}\left(j_{1}\right)$. For this reason, the relativization to $\exp ^{(4)}\left(2 j_{1}\right)$, which is necessary for technical reasons, does not essentially influence the sense of $\tau_{j, j_{1}}$ or $\tau_{j}\left(j_{1}\right)$. 
Let $\psi_{j}(z)$ stand for

$$
\begin{aligned}
\left(\exists y \in \mathcal{E}_{\exp } z\left\{\Gamma_{m}(y, \exp z)\right)\right. & \left.\wedge \bigwedge_{\Phi \leq j} \Gamma_{\Phi}(y, \exp z)\right) \\
& \left.\left.\wedge \forall l, l_{1}<z / 2 \forall x\left(x=\tau_{l, l_{1}} \Rightarrow \operatorname{True}(y, \exp z, x)\right)\right\}\right)^{\exp ^{(4)}(2 z)},
\end{aligned}
$$

where $x=\tau_{l, l_{1}}$ is an abbreviation for the inductive definition of $\tau_{l, l_{1}}$.

The following lemma establishes an important connection between $\tau_{j, j_{1}}$ and $\tau_{j}\left(j_{1}\right)$.

Lemma 3.1. Let $j \leq j_{1}$ and let $i$ be such that:

- The formulae $x=\tau_{l, l_{1}}$ (as a formula of $\left.x, l, l_{1}\right)$ and True $(y, \exp z, x)$ may be bounded by $\exp ^{(3)}(i)$ for any choice of $l, l_{1}<j_{1} / 2, z<j_{1}, y<$ $\exp \left(\pi\left(K\left(\exp j_{1}\right)\right)\right)$, and $x<K\left(\exp j_{1}\right)^{\exp j_{1}}$;

- $\Lambda_{i}$ is g.e. for $\left.\left\langle\varphi_{j, j_{1}}, \underline{j_{1}}\right\urcorner \bar{t}_{j_{1}}\right\rangle$ and for $\left\langle\psi_{j, j_{1}}, \underline{j_{1}}\right\rangle$.

Let $p \in \mathcal{E}_{i}$ satisfy $\Gamma_{m}(p, i)$. Then

$$
\mathbf{M}=\operatorname{True}\left(p, i, \tau_{j, j_{1}}\right) \quad \text { iff } \quad \mathbf{M}=\operatorname{True}\left(p, i, \tau_{j}\left(\underline{j_{1}}\right)\right) .
$$

REMARK 3.2. Any $i \geq \exp \left(2 j_{1}\right)$ satisfies the conditions of the lemma.

Proof. We prove the left-to-right direction as the other direction is very similar.

Assume $\mathbf{M} \models \operatorname{True}\left(p, i, \tau_{j, j_{1}}\right)$. As already noted, $\tau_{j, j_{1}}$ is $\varphi_{j, j_{1}}\left(j_{1}, \bar{t}_{j_{1}}\right)$. So, by the definition of $\tau_{j, j_{1}}$ and the meaning of the formula True, it follows that for all $l, l_{1}<j_{1} / 2$,

$$
p \models \operatorname{True}\left(s^{\varphi_{j, j_{1}}}\left(\underline{j_{1}} \bar{t}_{j_{1}}\right), \exp \underline{j_{1}}, \underline{\tau_{l, l_{1}}}\right) .
$$

We may assume that $m$ was chosen large enough so that the formula $x=\tau_{l, l_{1}}$ is $\exists_{m}$. Then, by our assumptions on the size of $i$, we may use the fact that $p$ satisfies $\Gamma_{m}$ to get $p=\left(\tau_{l, l_{1}}=\tau_{\underline{l}, l_{1}}\right)$ for all $l, l_{1}<j_{1} / 2$. Thus, for every $t$ such that $p \models\left(t=\tau_{\underline{l}, l_{\underline{l_{1}}}}\right)$, we also have $p \models\left(t=\underline{\tau_{l, l_{1}}}\right)$. By Definition 2.1, this means that

$$
p \models \forall x\left(x=\tau_{\underline{l}, \underline{l_{1}}} \Rightarrow \operatorname{True}\left(s^{\varphi_{j, j_{1}}}\left(\underline{j_{1}} \bar{t}_{j_{1}}\right), \exp \underline{j_{1}}, x\right)\right)
$$

for any choice of $l, l_{1}<j_{1} / 2$.

Similarly, for every $t$ such that $p \models\left(t<\underline{j_{1}} / 2\right)$, we also have $p \models(t=\underline{l})$ for some $l<j_{1} / 2$. Therefore, we get

$$
p=\forall l, l_{1}<\underline{j_{1}} / 2 \forall x\left(x=\tau_{l, l_{1}} \Rightarrow \operatorname{True}\left(s^{\varphi_{j, j_{1}}}\left(\underline{j_{1}} \frown \bar{t}_{j_{1}}\right), \exp \underline{j_{1}}, x\right)\right) .
$$

Combining this with the original assumption that $\mathbf{M} \models \operatorname{True}\left(p, i, \tau_{j, j_{1}}\right)$, we obtain 


$$
\begin{aligned}
& p \models\left(\left\{\Gamma_{m}\left(s^{\varphi_{j, j_{1}}}\left(\underline{j_{1}}\right\urcorner \bar{t}_{j_{1}}\right), \exp \underline{j_{1}}\right) \wedge \bigwedge_{\Phi \leq j} \Gamma_{\Phi}\left(s^{\varphi_{j, j_{1}}}\left(\underline{j_{1}}\right\urcorner \bar{t}_{j_{1}}\right), \exp \underline{j_{1}}\right) \\
& \left.\left.\wedge \forall l, l_{1}<\underline{j_{1}} / 2 \forall x\left(x=\tau_{l, l_{1}} \Rightarrow \operatorname{True}\left(s^{\varphi_{j, j_{1}}}\left(\underline{j_{1}} \bar{t}_{j}\right), \exp \underline{j_{1}}, x\right)\right)\right\}\right)^{\exp ^{(4)}\left(2 \underline{j_{1}}\right)} .
\end{aligned}
$$

To prove $\mathbf{M} \models \operatorname{True}\left(p, i, \tau_{j}\left(j_{1}\right)\right)$, we only need to check that $p$ also evaluates the above formula to "True" if we substitute the appropriate Skolem term for $s^{\varphi_{j, j_{1}}}\left(j_{1} \frown \bar{t}_{j_{1}}\right)$. If that was not the case, we would have neither $p \models \tau_{j}\left(\underline{j_{1}}\right)$ nor $\bar{p} \models \neg \tau_{j}\left(\underline{j_{1}}\right)$ (since we have a witness for the initial existential quantifier in $\left.\tau_{j}\left(\underline{j_{1}}\right)\right)$. But $p=\left(s_{\exp 2 j_{1}}=\exp ^{(4)}\left(2 \underline{j_{1}}\right)\right)$, so $p$ treats $\tau_{j}\left(\underline{j_{1}}\right)$ as a formula relativized to $s_{\exp 2 j_{1}}$. Now, $p \in \mathcal{E}_{i}$, and thus it follows from Definition 2.3(i) that at least one of $p \mid=\tau_{j}\left(\underline{j_{1}}\right)$ and $p=\neg \tau_{j}\left(\underline{j_{1}}\right)$ must hold.

Corollary 3.3. Let $j \leq j_{1}$. Then

$$
\mathbf{M} \models \forall i\left(\Lambda_{i} \text { g.e. for }\left\langle\varphi_{j, j_{1}}, \underline{j_{1}} \frown \bar{t}_{j_{1}}\right\rangle \Rightarrow \exists p \in \mathcal{E}_{i} \operatorname{True}\left(p, i, \tau_{j, j_{1}}\right)\right)
$$

iff

$$
\mathbf{M}=\forall i\left(\Lambda_{i} \text { g.e. for }\left\langle\psi_{j}, \underline{j_{1}}\right\rangle \Rightarrow \exists p \in \mathcal{E}_{i} \operatorname{True}\left(p, i, \tau_{j}\left(\underline{j_{1}}\right)\right) .\right.
$$

Proof. Follows from the lemma via Propositions 2.5 and 2.6.

We now let $\tau$ be $\forall j \forall j_{1} \operatorname{Sat}\left(\tau_{j}\left(j_{1}\right)\right)$. In view of Lemma 3.1, the sentence $\tau$ can be treated as a form of reflection principle (an observation due to A. Blass). Indeed, a " $\Pi_{1}$ reflection principle" is usually understood to be a formalized version of the principle

$$
\psi \text { is provable } \Rightarrow \psi \text { is true, }
$$

for $\psi \in \Pi_{1}$, in other words,

$$
\phi \text { is true } \Rightarrow \phi \text { is consistent, }
$$

for $\phi \in \Sigma_{1}$. Now, the existence of evaluations which satisfy $\phi$ is a kind of consistency of $\phi$. So, in any model in which Sat is well-behaved as a truth definition, $\tau$ says:

$\phi$ is true $\Rightarrow \phi$ plus a restricted fragment of $\tau$ is consistent,

for $\phi \in \Sigma_{1}$. Thus, $\tau$ expresses $(* *)$ and additionally has a limited "selfreproducing" property.

As remarked above, $\tau$ is a consistent sentence. Even more:

TheOREM 3.4. The theory $I \Delta_{0}+\exp$ proves $\tau$.

Proof. Let us work in a model of $I \Delta_{0}+\exp$. We prove $\forall j \leq j_{1} \operatorname{Sat}\left(\tau_{j}\left(j_{1}\right)\right)$ by induction on $j_{1}$. This will suffice, as for any $j$ and any $j_{1}^{\prime}<j_{1}, \tau_{j}\left(j_{1}\right)$ implies $\tau_{j}\left(j_{1}^{\prime}\right)$ thanks to Propositions 2.5 and 2.6. 
Assume $\forall j \leq j_{1} \operatorname{Sat}\left(\tau_{j}\left(j_{1}\right)\right)$. We want to show $\forall j \leq j_{1}+1 \operatorname{Sat}\left(\tau_{j}\left(j_{1}\right)\right)$. Thus, given any $j \leq j_{1}+1$, we need

$$
\left(\exists p \in \mathcal { E } _ { \operatorname { e x p } ( j _ { 1 } + 1 ) } \left\{\Gamma_{m}\left(p, \exp \left(j_{1}+1\right)\right) \wedge \bigwedge_{\Phi \leq j} \Gamma_{\Phi}\left(p, \exp \left(j_{1}+1\right)\right)\right.\right.
$$

$\left.\left.\wedge \forall l, l_{1}<\left(j_{1}+1\right) / 2 \forall x\left(x=\tau_{l, l_{1}} \Rightarrow \operatorname{True}\left(p, \exp \left(j_{1}+1\right), x\right)\right)\right\}\right)^{\exp ^{(4)}\left(2\left(j_{1}+1\right)\right)}$.

We will find an evaluation $p$ of rank $j_{1}+1$ such that

$$
\begin{aligned}
\Gamma_{m}\left(p, \exp \left(j_{1}+1\right)\right) \wedge & \bigwedge_{\Phi \leq j} \Gamma_{\Phi}\left(p, \exp \left(j_{1}+1\right)\right) \\
& \wedge \forall l, l_{1}<\left(j_{1}+1\right) / 2 \operatorname{True}\left(p, \exp \left(j_{1}+1\right), \tau_{l}\left(\underline{l_{1}}\right)\right) .
\end{aligned}
$$

The fact that $p$ is as required in $\tau_{j}\left(j_{1}+1\right)$ will then follow from Lemma 3.1, since $\exp \left(j_{1}+1\right)$ is a large enough rank for the lemma to ensure the equivalence of $p \models \tau_{l, l_{1}}$ and $p \models \tau_{l}\left(\underline{l_{1}}\right)$ for $l \leq l_{1}<\left(j_{1}+1\right) / 2$ (see Remark 3.2).

The way to obtain $p$ is by constructing a Skolem hull on $\Lambda_{\exp \left(j_{1}+1\right)}$. A Skolem hull on a given $\Lambda$ is a sequence $H=\left\langle h_{t}: t \in \Lambda\right\rangle$ of elements of $M$, where the element $h_{t}$ is thought of as an interpretation of the term $t$. One may define the satisfaction relation $H=\varphi(\bar{t})$ in much the same way as $p \models \varphi(\bar{t})$, i.e. by postulating

$$
\text { - } H=\varphi(\bar{t}) \text { iff } \mathbf{M}=\varphi\left(\bar{h}_{t}\right)
$$

for $\varphi$ open, and then proceeding as in Definition 2.1, so that e.g.

- $H=\exists y \varphi^{\prime}(\bar{t}, y)$ iff $\Lambda_{i}$ is g.e. for $\left\langle\exists y \varphi^{\prime}(\bar{x}, y), \bar{t}\right\rangle$ and $H=\varphi^{\prime}\left(\bar{h}_{t}, h_{s^{\varphi}(\bar{t})}\right)$.

It is clear that any hull $H$ on $\Lambda$ determines an evaluation $p_{H}$ such that $p_{H}=\varphi$ iff $H \models \varphi$. If $\Lambda$ is $\Lambda_{i}$, and $H$ is a hull of rank $i$ (defined analogously to "evaluation of rank $i$ ", cf. Def. 2.3), then $p_{H} \in \mathcal{E}_{i}$.

The hull we want to construct on $\Lambda_{\exp \left(j_{1}+1\right)}$ is to satisfy:

(i) for any $h \in H, h \leq \exp ^{(4)}\left(j_{1}+1\right)$,

(ii) for any $a \leq K\left(\exp j_{1}\right), h_{\underline{a}}=a$, and for any $j^{\prime} \leq \exp \left(j_{1}+1\right)$, $h_{s_{j^{\prime}}}=\exp ^{(3)}\left(j^{\prime}\right)$;

(iii) for any $h_{t} \in H$ and for any formula of the form $\psi^{t}$ smaller than $\exp \left(\exp \left(j_{1}+1\right)-1\right)$,

$$
\left(H=\psi^{t}\right) \quad \text { iff } \quad \operatorname{Sat}\left(\psi^{h_{t}}\right) ;
$$

(iv) for any $h_{t} \in H$ and for any formula of the form $\exists x \leq t \psi^{x}$ smaller than $\exp \left(\exp \left(j_{1}+1\right)-1\right)$,

$$
\left(H \models \exists x \leq t \psi^{x}\right) \quad \text { iff } \quad \operatorname{Sat}\left(\exists x \leq h_{t} \psi^{x}\right) ;
$$

(v) for every $\varphi<\exp \left(j_{1}+1\right)$,

$$
\text { if } \operatorname{Sat}\left(\forall x \leq \exp ^{(4)}\left(j_{1}+1\right) \neg \phi^{x}\right) \text { then } H \models \forall x \neg \varphi^{x} .
$$


The actual construction of $H$ is based on a straightforward induction. Given that $\Lambda_{\exp \left(j_{1}+1\right)}$ is ordered as $\left\langle t_{1}, \ldots, t_{k}\right\rangle$, we assign interpretations to the $t_{r}$ 's by induction on $r \leq k$. If $t_{r+1}$ is $\underline{a}$, then $h_{t_{r+1}}=a$, if it is $s_{j^{\prime}}$, then $h_{t_{r+1}}=\exp ^{(3)} j^{\prime}$. If $t_{r+1}=s^{\varphi}(\bar{t})$, then we define $h_{t_{r+1}}$ to be the smallest witness below $\exp ^{(4)}\left(j_{1}+1\right)$ for $\varphi\left(\bar{h}_{t}\right)$ whenever it exists, and arbitrary (but smaller than $\left.\exp ^{(4)}\left(j_{1}+1\right)\right)$ if there is no such witness.

We take $p$ to be $p_{H}$. It is again straightforward to check that $p \in$ $\mathcal{E}_{\exp \left(j_{1}+1\right)}$ and that $p$ has all the properties required in $(*)$. In particular, $\forall l, l_{1}<\left(j_{1}+1\right) / 2 \operatorname{True}\left(p, \exp \left(j_{1}+1\right), \tau_{l}\left(\underline{l_{1}}\right)\right)$ follows by the construction of $p$ from the inductive assumption $\forall j \leq j_{1}$ Sat $\left(\tau_{j}\left(j_{1}\right)\right)$.

4. The main theorem. To define the theory $T$ mentioned in the introduction, we will use "finite fragments" of the principle $\tau$. Namely, let $\tau_{n}$ denote

$$
\begin{aligned}
\forall i \exists p \in \mathcal{E}_{i}\left(\left\{\Gamma_{m}(p, i)\right.\right. & \wedge \bigwedge_{\Phi \leq n} \Gamma_{\Phi}(p, i) \\
& \left.\left.\wedge \forall l, l_{1}<(\log i) / 2 \forall x\left(x=\tau_{l, l_{1}} \Rightarrow \operatorname{True}(p, i, x)\right)\right\}\right)^{\exp ^{(3)}(2 \log i)} .
\end{aligned}
$$

In the notation of the previous section, $\tau_{n}$ is (approximately) $\forall j_{1} \tau_{n}\left(j_{1}\right)$. In particular, for $n \in \omega, \tau_{n}$ is a standard $\Pi_{1}$ sentence.

LEMMA 4.1. Any $\exists_{m}$ sentence $\chi$ consistent with $I \Delta_{0}+\Omega_{3}$ is consistent with all the $\tau_{n}$ 's.

Proof. Let $\mathbf{M} \models I \Delta_{0}+\Omega_{3}+\tau_{0}+\chi$. We may assume that $\mathbf{M} \models B \Sigma_{1}$, since (cf. e.g. $[\mathrm{P}]) \mathbf{M}$ has a 1-elementary extension $\mathbf{M}^{\prime}$ of the same height satisfying $B \Sigma_{1}$.

Let $\mathcal{F}$ be the set

$$
\left\{\tau_{n}(x): n \in \omega\right\} \cup\{\chi\}
$$

This is a subset of

$$
\left\{\tau_{l}(x): l \in \log ^{(4)}\right\} \cup\{\chi\} .
$$

Using the fact that $\mathbf{M}=\tau_{0}$, we infer from (a minor variant of) Corollary 3.3 that

$$
\forall i \exists p \in \mathcal{E}_{i} \forall l, j_{1}<(\log i) / 2 p \models \tau_{l}\left(\underline{j_{1}}\right),
$$

since $\Lambda_{i}$ is g.e. for $\left\langle\psi_{l}, j_{1}\right\rangle$ whenever $l, j_{1}<(\log i) / 2$. Also, almost all the $p$ 's evaluate $\chi$ to "True", because all $p$ 's given by $\tau_{0}$ satisfy $\Gamma_{m}$, and there is an $i$ such that a witness for $\chi$ exists below $\exp ^{(3)}(i)$. Since $\log ^{(4)}$ is closed under addition, we may apply Theorem 2.11 and obtain an increasing and cofinal sequence $P_{0}$ of evaluations such that: $\mathbf{M}\left[P_{0}\right] \models I \Delta_{0}+\Omega_{3}, P_{0} \models \chi$, and $P_{0}=\tau_{n}\left(l_{1}\right)$ for any $n \in \omega$ and $j_{1} \in \log ^{(4)}(\mathbf{M})$. Since the $\tau_{n}(\cdot)$ 's are $\Pi_{1}$, it follows that $\mathbf{M}\left[P_{0}\right] \models \tau_{n}\left(j_{1}\right)$ for any $n$ and $j_{1}$. 
But this means that for any $n, j_{1}$,

$$
\begin{aligned}
\mathbf{M}\left[P_{0}\right] \models \exists p \in \mathcal{E}_{\exp j_{1}}\left\{\Gamma_{m}\left(p, \exp j_{1}\right) \wedge \bigwedge_{\Phi \leq n} \Gamma_{\Phi}\left(p, \exp j_{1}\right)\right. \\
\left.\wedge \forall l, l_{1}<j_{1} / 2 \forall x\left(x=\tau_{l, l_{1}} \Rightarrow \operatorname{True}\left(p, \exp j_{1}, x\right)\right)\right\} .
\end{aligned}
$$

We may obtain suitable $p$ 's in $\mathcal{E}_{i}$ for $i$ not of the form $\exp j_{1}$ by restricting the evaluations we have in $\mathcal{E}_{\exp j_{1}}$ (use Propositions 2.6 and 2.5 to ensure that these restrictions are indeed evaluations of appropriate ranks and that they have the desired properties). Hence, for any $n$ we see that, in $\mathbf{M}\left[P_{0}\right]$,

$$
\begin{aligned}
\forall i \exists p \in \mathcal{E}_{i}\left\{\Gamma_{m}(p, i)\right. & \wedge \bigwedge_{\Phi \leq n} \Gamma_{\Phi}(p, i) \\
& \left.\wedge \forall l, l_{1}<(\log i) / 2 \forall x\left(x=\tau_{l, l_{1}} \Rightarrow \operatorname{True}(p, i, x)\right)\right\},
\end{aligned}
$$

which implies that $\mathbf{M} \models \tau_{n}$ for all $n$. It remains to point out that $\mathbf{M}\left[P_{0}\right]$ also satisfies $\chi$, since $P_{0} \models \chi$ and we may in this context treat $\chi$ as a $\Delta_{0}$ formula by considering its relativization to the smallest witness for $\chi$.

Observe that the construction described in the proof of the lemma would have also worked if we started in a model of some higher $\tau_{N}$, and not just $\tau_{0}$. In that case, we would be able to replace the set $\mathcal{F}$ by a set which additionally contains $\neg \Phi$ for all $\Phi \leq N$ false in $\mathbf{M}$, and suitable true relativizations of $\Phi$ for $\Phi \leq N$ true in $\mathbf{M}$. Theorem 2.11 would then give us a sequence $P_{N}$ corresponding to that set. $\mathbf{M}\left[P_{N}\right]$ would satisfy all the $\tau_{n}$ 's and $\chi$ just as $\mathbf{M}\left[P_{0}\right]$ did, but it would additionally satisfy exactly those $\Phi \leq N$ which are true in $\mathbf{M}$.

Given any $\Pi_{1}$ consequence $\theta$ of $I \Delta_{0}+\exp$, there exists a purely existential sentence $\varphi_{\theta}$ consistent with $I \Delta_{0}+\Omega_{3}+B \Sigma_{1}+\theta$, but inconsistent with $I \Delta_{0}+\exp ($ see $[\mathrm{HP}])$. If we apply this result to $\theta:=\tau_{0}$, we obtain an $\exists_{m}$ sentence $\chi$ consistent with $I \Delta_{0}+\Omega_{3}+B \Sigma_{1}+\left\{\tau_{n}: n \in \omega\right\}$, but inconsistent with $I \Delta_{0}+$ exp. Fix such a $\chi$ and define the theory $T_{0}$ by

$$
T_{0}:=I \Delta_{0}+\Omega_{3}+B \Sigma_{1}+\left\{\tau_{n}: n \in \omega\right\}+\chi .
$$

In what follows, $\varepsilon$ will denote a binary sequence, $\varepsilon=\left\langle\varepsilon_{0}, \ldots, \varepsilon_{\operatorname{lh}(\varepsilon)-1}\right\rangle$.

Given a fixed $\Delta_{0}$ enumeration $\left\langle\varphi_{n}: n \in \omega\right\rangle$ of all $\Sigma_{1}$ sentences, let us introduce the sentences

$$
\begin{aligned}
\sigma_{n, \varepsilon} & :=(n=\operatorname{lh}(\varepsilon)) \\
\wedge \forall i \exists p \in \mathcal{E}_{i}\left(\Gamma_{m}(p, i) \wedge \forall l, l_{1}\right. & <(\log i) / 2 \forall x\left(x=\tau_{l, l_{1}} \Rightarrow \operatorname{True}(p, i, x)\right) \\
& \left.\wedge \forall k<n\left(\varepsilon_{k}=0 \Rightarrow p=\neg \varphi_{k}\right) \wedge p=\neg \varphi_{n}\right) .
\end{aligned}
$$

Lemma 4.2. Assuming $m$ is sufficiently large, for any $n$ and $\varepsilon, \sigma_{n, \varepsilon}$ is (equivalent in $I \Delta_{0}+\Omega_{3}$ to) $a \forall_{m}$ sentence. 
Proof. The only difficulty is to show that $\forall i \exists p \in \mathcal{E}_{i} \Gamma_{m}(p, i)$ can be equivalently written as a $\forall_{m}$ formula. $\forall i \exists p \in \mathcal{E}_{i} \Gamma_{m}(p, i)$ is

$$
\begin{aligned}
\forall i \exists p \in \mathcal{E}_{i} \forall j \leq i \forall \psi<i, \psi \in \exists_{m} \forall \underline{a_{1}}, \ldots, \underline{a_{r}} \in \Lambda_{i} \\
\quad\left(\operatorname{Sat}_{m}\left(\psi^{\exp ^{(3)}(j)}\left(a_{1}, \ldots, a_{r}\right)\right) \Rightarrow \operatorname{True}\left(p, i, \psi^{s_{j}}\left(\underline{a}_{1}, \ldots, \underline{a}_{r}\right)\right)\right),
\end{aligned}
$$

so the main problem is that an implication with the $\exists_{m}$ precedent Sat $_{m}$ occurs in the scope of the bounded existential quantifier $\exists p$. Actually, we could clearly replace $\mathrm{Sat}_{m}$ by its $E_{m}$ analogue $\operatorname{Sat}_{E_{m}}$ (see Preliminaries), but this still does not solve our problem.

By the definition of the class $\exists_{m}$, an $\exists_{m}$ formula $\psi\left(a_{1}, \ldots, a_{r}\right)$ is $\exists x \psi^{\prime}\left(a_{1}, \ldots, a_{r}, x\right)$ for $\psi^{\prime} \in U_{m-1}$. Thus $\psi^{\exp ^{(3)}(j)}\left(a_{1}, \ldots, a_{r}\right)$ holds iff there is a witness $x \leq \exp ^{(3)}(j)$ such that $\psi^{\prime}\left(a_{1}, \ldots, a_{r}, x\right)$.

Consider the sentence $\xi_{m}$ given by

$$
\begin{aligned}
& \forall i \forall\left\langle x_{j, \psi, \underline{\bar{a}}}: j \leq i, \psi<i \text { in } \exists_{m}, \underline{\bar{a}} \in \Lambda_{i} \text { of appropriate length }\right\rangle \\
& \text { such that each } x_{j, \psi, \underline{a}} \text { is } \leq \exp ^{(3)}(j) \\
& \exists p \in \mathcal{E}_{i} \forall j \leq i \forall \psi<i, \psi \in \exists_{m} \forall \underline{a_{1}}, \ldots, \underline{a_{r}} \in \Lambda_{i} \\
& \quad\left(\operatorname{Sat}_{U_{m-1}}\left(\psi^{\prime}\left(a_{1}, \ldots, a_{r}, x_{j, \psi, \underline{a}}\right)\right) \Rightarrow \operatorname{True}\left(p, i, \psi^{s_{j}}\left(\underline{a}_{1}, \ldots, \underline{a}_{r}\right)\right)\right),
\end{aligned}
$$

where $\left\langle x_{j, \psi, \underline{a}}\right\rangle$ should be thought of as a sequence of "potential witnesses" smaller than $\exp ^{(3)}(j)$ for $\psi(\underline{a})$, and $\operatorname{Sat}_{U_{m-1}}$ is dual to $\operatorname{Sat}_{E_{m-1}}$.

$\xi_{m}$ is easily seen to be equivalent to a $\forall_{m}$ sentence. Indeed: Sat $U_{m-1}$ is $U_{m-1}$ with an appropriately large parameter, so it is $E_{m-1}$ in the antecedent of an implication; the universal quantifiers for $j, \psi$, and $\underline{\bar{a}}$ may be treated as sharply bounded (in particular, $\bar{a}$ is an at most $\log \psi$-long sequence of objects smaller than $3 K(i-1)$, so it is $\left.\leq(3 K(i-1))^{\log i} \in \log \right)$; and the initial unbounded universal quantifiers may obviously be merged into one.

Moreover, $\xi_{m}$ is also equivalent to $\forall i \exists p \in \mathcal{E}_{i} \Gamma_{m}(p, i)$. The right-to-left direction is trivial: for any $i$ the $p \in \mathcal{E}_{i}$ satisfying $\Gamma(p, i)$ will be good for all sequences of witnesses. For the other direction, given a fixed $i$, there is always an "optimal" sequence of witnesses $\left\langle x_{j, \psi, \underline{a}}\right\rangle$, i.e. one such that if there is any $x \leq \exp ^{(3)} j$ for which $\psi^{\prime}\left(a_{1}, \ldots, a_{r}, x\right)$ holds, then $x_{j, \psi, \underline{\underline{a}}}$ is such an $x$. Now, $\xi_{m}$ gives us a $p \in \mathcal{E}_{i}$ which works for this "optimal" sequence. One easily checks that $p$ must satisfy $\Gamma_{m}(p, i)$.

We also introduce the sentences $\Psi_{n}$, for $n \in \omega$ :

$$
\Psi_{n}:=\bigvee_{\varepsilon \in\{0,1\}^{n+1}}\left(\bigwedge_{r \leq n, \varepsilon_{r}=0} \neg \phi_{r} \wedge \bigwedge_{r \leq n, \varepsilon_{r}=1}\left(\phi_{r} \wedge \neg \sigma_{r,(\varepsilon \mid r)}\right)\right) .
$$

Finally, we define our theory $T$ by

$$
T:=T_{0}+\left\{\Psi_{n}: n \in \omega\right\} .
$$


Obviously, $T$ is a recursive theory. We will now prove our main theorem, which shows, among other things, that $T$ axiomatizes a certain class of models of $T_{0}$ in which the set of elements definable by $\Delta_{0}$ formulae of restricted complexity is cofinal in the set of all $\Delta_{0}$ definable elements:

TheOREM 4.3. (a) $T$ is consistent.

(b) For any (not necessarily countable) $\mathbf{M}=T, \mathbf{M}^{\Sigma_{1}}$ is recursively reducible to $\mathbf{M}^{\exists}$.

(c) In any (not necessarily countable) $\mathbf{M} \models T, E_{m+1} \wedge U_{m+1}(\mathbf{M})$ is cofinal in $\Delta_{0}(\mathbf{M})$.

Proof. We first prove (a). The proof is an inductive construction based on repeated application of Theorem 2.11.

In the initial step, take an arbitrary countable model $\mathbf{M}$ of $T_{0}$. Consider $\sigma_{0}=\sigma_{0, \emptyset}$ and put

$$
\mathbf{M}_{0}^{\prime}= \begin{cases}\mathbf{M}\left[P_{0}\right] & \text { if } \mathbf{M}=\sigma_{0}, \\ \mathbf{M} & \text { otherwise, }\end{cases}
$$

where $P_{0}$ is as in Theorem 2.11 for $k=1$ and $\mathcal{F}_{0}$ is defined as

$$
\left\{\tau_{n}(x): n \in \omega\right\} \cup\left\{\neg \phi_{0}\right\} \cup\{\chi\},
$$

where $\chi$ should be treated as a relativization of the original $\chi$ to some $\exp ^{(3)}(\cdot)$ true in $\mathbf{M}$ (note that the existence of $P_{0}$ follows from the fact that $\mathbf{M}=\sigma_{0}$ via Theorem 2.11 and Lemma 3.1). Also let $\varepsilon_{0}=0$ in the former and $\varepsilon_{0}=1$ in the latter case.

$\mathbf{M}_{0}^{\prime}$ clearly satisfies $I \Delta_{0}+\Omega_{3}$ and $\left\{\tau_{n}: n \in \omega\right\}+\chi$ (either by our assumptions on $\mathbf{M}$ or by the choice of $\mathcal{F}_{0}$ ). Furthermore, by Lemma 2.10, $\mathbf{M} \models \sigma_{0}$ implies $\mathbf{M}\left[P_{0}\right] \models \neg \phi_{0}$. On the other hand, in all models of $\left\{\tau_{n}\right.$ : $n \in \omega\}, \neg \phi_{0}$ implies $\sigma_{0}$, because of the validity of a suitable $\tau_{N}$. Hence either

$$
\varepsilon_{0}=0 \quad \text { and } \quad \mathbf{M}_{0}^{\prime}=\neg \phi_{0}
$$

or

$$
\varepsilon_{0}=1 \quad \text { and } \quad \mathbf{M}_{0}^{\prime}=\phi_{0} \wedge \neg \sigma_{0} .
$$

In other words, $\mathbf{M}_{0}^{\prime} \models \Psi_{0}$. Thus, we always have $\mathbf{M}_{0}^{\prime}=\left(T_{0} \backslash B \Sigma_{1}\right)+\Psi_{0}$. By passing to a 1-elementary extension of the same height if necessary (see the beginning of the proof of Lemma 4.1), we may obtain a model $\mathbf{M}_{0}$ satisfying $T_{0}+\Psi_{0}$.

Proceeding inductively, assume that we are given a model $\mathbf{M}_{n}$ satisfying $T_{0}+\Psi_{n}$. As in the initial step, consider $\sigma_{n+1}=\sigma_{n+1, \varepsilon}$ for the sequence $\varepsilon=\left\langle\varepsilon_{0}, \ldots, \varepsilon_{n}\right\rangle$ determined uniquely in view of $\mathbf{M}_{n} \models \Psi_{n}$. Put

$$
\mathbf{M}_{n+1}^{\prime}= \begin{cases}\mathbf{M}_{n}\left[P_{n+1}\right] & \text { if } \mathbf{M}=\sigma_{n+1}, \\ \mathbf{M}_{n} & \text { otherwise }\end{cases}
$$


where $P_{n+1}$ is as in Theorem 2.11 for $k=1$ and $\mathcal{F}_{n+1}$ defined as

$$
\begin{aligned}
& \left\{\tau_{n}(x): n \in \omega\right\} \cup\left\{\neg \phi_{r}: r \leq n, \varepsilon_{r}=0\right\} \\
& \cup\left\{\neg \sigma_{r,(\varepsilon \mid r)}: r \leq n, \varepsilon_{r}=1\right\} \cup\left\{\neg \phi_{n+1}\right\} \cup\{\chi\},
\end{aligned}
$$

where $\chi$ and the $\neg \sigma$ 's should again be treated as true relativizations to some $\exp ^{(3)}(\cdot)$ (note as previously that the existence of $P_{n+1}$ follows from $\mathbf{M}_{n}=\sigma_{n+1}$ via Theorem 2.11 and Lemma 3.1). Define $\varepsilon_{n+1}=0$ in the former and $\varepsilon_{n+1}=1$ in the latter case.

Again, it is clear that $\mathbf{M}_{n+1}^{\prime}$ satisfies $I \Delta_{0}+\Omega_{3}$ and $\left\{\tau_{n}: n \in \omega\right\}+\chi$. As in the initial step, we get either

$$
\varepsilon_{n+1}=0 \quad \text { and } \quad \mathbf{M}_{n+1}^{\prime} \models \neg \phi_{n+1}
$$

or

$$
\varepsilon_{n+1}=1 \quad \text { and } \quad \mathbf{M}_{n+1}^{\prime} \mid=\phi_{n+1} \wedge \neg \sigma_{n+1} .
$$

We now check that $\mathbf{M}_{n+1}^{\prime} \models \Psi_{n+1}$. This is obvious if $\varepsilon_{n+1}=1$, so assume $\varepsilon_{n+1}=0$ and thus $\mathbf{M}_{n+1}^{\prime}=\mathbf{M}_{n}\left[P_{n+1}\right]$. For a given $r \leq n$, if $\varepsilon_{r}=0$, then $\mathbf{M}_{n+1}^{\prime}=\neg \phi_{r}$ as required, since $P_{n+1} \mid=\neg \phi_{r}$. On the other hand, if $\varepsilon_{r}=1$, then $\mathbf{M}_{n+1}^{\prime} \models \neg \sigma_{r,(\varepsilon \mid r)}$, since $P_{n+1}$ sets a suitable relativization of $\neg \sigma_{r,(\varepsilon \mid r)}$ to "True". But this also means $\mathbf{M}_{n+1}^{\prime} \models \phi_{r}$, as $\neg \phi_{r}$ would imply $\sigma_{r,(\varepsilon \mid r)}$ in view of a suitable $\tau_{N}$. Thus, in either case, $\mathbf{M}_{n+1}^{\prime} \mid=\Psi_{n+1}$.

As before, we may pass to a 1-elementary extension if necessary to get a model $\mathbf{M}_{n+1}$ satisfying $T_{0}+\Psi_{n}$. Since $\Psi_{n}$ clearly implies $\Psi_{k}$ for $k<n$, this shows that every finite subtheory of $T$ is consistent. By compactness, $T$ itself is also consistent, which ends the proof of (a).

To prove (b), let $\mathbf{M}$ be an arbitrary model of $T$. Let the infinite binary sequence $\varepsilon$ be the unique extension of the sequences given by the $\Psi_{n}$ 's. Then for each $n \in \omega$ we have

$$
\mathbf{M} \models \phi_{n} \equiv \neg \sigma_{n,(\varepsilon\lceil n)} .
$$

For, just as in the proof of (a), $\neg \phi_{n}$ implies $\sigma_{n,(\varepsilon\lceil n)}$ since $\mathbf{M} \models\left\{\tau_{n}: n \in \omega\right\}$, while $\phi_{n}$ yields $\varepsilon_{n}=1$, whence we have $\neg \sigma_{n,(\varepsilon \mid n)}$ because of $\Psi_{n}$.

From $(*)$ we obtain a recursive reduction of $\Sigma_{1}$ truth about $\mathbf{M}$ to $\exists_{m}$ truth about $\mathbf{M}$. Indeed, knowing $\left(\varepsilon\lceil n)\right.$ and knowing whether $\sigma_{n,(\varepsilon\lceil n)}$ is true we deduce whether $\phi_{n}$ is true, whence we deduce $(\varepsilon\lceil n+1)$ and so on: we recover the $\Sigma_{1}$ truth from the $\forall_{m}$ truth step by step.

For the proof of (c), suppose that $a \in \Delta_{0}(\mathbf{M})$. In other words, $\mathbf{M}=\varphi(a)$, where $\varphi(x) \in \Delta_{0}$ and $\mathbf{M}=\exists ! x \varphi^{x}(x)$. Thus, $\exists x \varphi^{x}(x)$ is a $\Sigma_{1}$ sentence, say $\phi_{n}$, true in $\mathbf{M}$. Let $i$ be such that $\exp ^{(3)}(i)<a$, so that we have $\mathbf{M}=\neg \exists x<$ $\exp ^{(3)}(i) \varphi^{x}(x)$. Using an appropriate $\tau_{N}$ (recall that $\left.\mathbf{M} \models\left\{\tau_{n}: n \in \omega\right\}\right)$, we can find a $p \in \mathcal{E}_{i}$ such that in $\mathbf{M}$ we have 


$$
\begin{aligned}
\Gamma_{m}(p, i) & \wedge \forall l, l_{1}<(\log i) / 2 \forall x\left(x=\tau_{l, l_{1}} \Rightarrow \operatorname{True}(p, i, x)\right) \\
& \wedge \bigwedge_{r<n}\left(\varepsilon_{r}=0 \Rightarrow p=\neg \phi_{r}\right) \wedge p=\neg \phi_{n},
\end{aligned}
$$

where $\varepsilon$ is the sequence given by $\Psi_{n}$.

Hence

$$
\mathbf{M} \models \bar{\sigma}_{n, \varepsilon}(i),
$$

where $\bar{\sigma}_{n, \varepsilon}$ is obtained from $\sigma_{n, \varepsilon}$ (the standard version, not necessarily the one discussed in Lemma 4.2) by deleting the universal quantifier $\forall i$.

We have proved that for any $i, \neg \exists x<\exp ^{(3)}(i) \varphi^{x}(x)$ implies $\bar{\sigma}_{n, \varepsilon}(i)$. However, we have $\mathbf{M} \models \neg \sigma_{n, \varepsilon}$ since $\mathbf{M} \models \phi_{n}$. It follows from $\neg \sigma_{n, \varepsilon}$ that there exists a number $i_{0}$ such that $\mathbf{M} \models \neg \bar{\sigma}_{n, \varepsilon}\left(i_{0}\right)$.

Let $b_{0}$ be $\exp ^{(4)}\left(2 \cdot \log i_{0}\right)$ (thus, $b_{0}$ is large enough to be a bound for all the quantifiers in $\left.\bar{\sigma}_{n, \varepsilon}\left(i_{0}\right)\right)$, and let $b>b_{0}$ be the smallest element of $\mathbf{M}$ which is large enough to be a bound for all the quantifiers in $b_{0}=\exp ^{(4)}\left(2 \cdot \log i_{0}\right)$. Now, $b$ is the smallest element satisfying the $E_{m+1}$ formula

$$
\exists i_{0}<b \exists b_{0}<b\left(\left(b_{0}=\exp ^{(4)}\left(2 \cdot \log i_{0}\right)\right)^{b} \wedge \neg\left(\bar{\sigma}_{n, \varepsilon}\left(i_{0}\right)\right)^{b_{0}}\right),
$$

so it is definable in $\mathbf{M}$ by an $E_{m+1} \wedge U_{m+1}$ formula. Furthermore, $b>a$. This proves that (c) holds.

We conclude this paper with a remark on $\Sigma_{1}$ definability of $\mathbb{N}$ in models of $I \Delta_{0}+\Omega_{1}$-more precisely, on its relation to the question whether elements definable by $\Sigma_{1}$ formulae of some fixed complexity are cofinal in a given model.

Let $\mathbf{M}=I \Delta_{0}+\Omega_{1}$ and assume that the set $\exists_{r}(\mathbf{M})$ is cofinal in $\mathbf{M}$. We claim that if $\mathbf{M}^{\exists_{r}}$ has a code $a \in \mathbf{M}$, then $\mathbb{N}$ is $\Sigma_{1}$ definable in $\mathbf{M}$ with $a$ as a parameter. For, given an enumeration $\left\langle\varphi_{n}: n \in \mathbb{N}\right\rangle$ of $\exists_{r}$ sentences, let $\psi(x)$ be the formula

$$
\exists y \forall z<x\left(\varphi_{z} \in a \Rightarrow \operatorname{Sat}_{r}^{y}\left(\varphi_{z}\right)\right) .
$$

Clearly, it follows from the cofinality of $\exists_{r}(\mathbf{M})$ that $\psi(x)$ defines $\mathbb{N}$ in $\mathbf{M}$.

We have thus proved one half of the following proposition (the other follows easily by a standard argument):

Proposition 4.4. Assume that $\mathbb{N}$ is not $\Sigma_{1}$ definable (with parameters) in $\mathbf{M}$. Then for any $r: \exists_{r}(\mathbf{M})$ is cofinal in $\mathbf{M}$ iff $\exists_{r}$ truth is not codable in $\mathbf{M}$.

\section{References}

[A1] Z. Adamowicz, A contribution to the end-extension problem and the $\Pi_{1}$ conservativeness problem, Ann. Pure Appl. Logic 61 (1993), 3-48.

[A2] -, On tableau consistency in weak theories, preprint 618, Inst. Math., Polish Acad. Sci., July 2001. 
[A3] Z. Adamowicz, Herbrand consistency and bounded arithmetic, Fund. Math. 171 (2002), 279-292.

[AZ1] Z. Adamowicz and P. Zbierski, On Herbrand type consistency in weak theories, Arch. Math. Logic 40 (2001), 399-413.

[AZ2] -, 一, On complexity reduction of $\Sigma_{1}$ formulas, ibid. 42 (2003), 45-58.

[HP] P. Hájek and P. Pudlák, Metamathematics of First Order Arithmetic, Springer, Berlin, 1993.

[P] J. B. Paris, Some conservation results for fragments of arithmetic, in: Model Theory for Algebra and Arithmetic, Lecture Notes in Math. 890, Springer, 1981, 251-262.

[S] S. Salehi, Herbrand consistency in arithmetics with bounded induction, Ph.D. thesis, Inst. Math., Polish Acad. Sci., 2002.

Zofia Adamowicz

Institute of Mathematics

Polish Academy of Sciences

Śniadeckich 8

00-956 Warszawa, Poland

E-mail: zosiaa@impan.gov.pl
Leszek Aleksander Kołodziejczyk Institute of Philosophy Warsaw University Krakowskie Przedmieście 3 00-047 Warszawa, Poland E-mail: 1.kolodziejczyk@zodiac.mimuw.edu.pl

Received 4 March 2002;

in revised form 22 October 2002 and 2 December 2003 